\title{
Interactive comment on "Ice multiplication from ice-ice collisions in the high Arctic: sensitivity to ice habit, rimed fraction and the spectral representation of the colliding particles" by Georgia Sotiropoulou et al.
}

Anonymous Referee \#3

Received and published: 8 January 2021

My review is in the attached supplement as a PDF.

Please also note the supplement to this comment:

https://acp.copernicus.org/preprints/acp-2020-786/acp-2020-786-RC2-

supplement.pdf 